CHRONIC OBSTRUCTIVE PULMONARY DISEASE

\title{
Functional variants of antioxidant genes in smokers with COPD and in those with normal lung function
}

\author{
R P Young, R Hopkins, P N Black, C Eddy, L Wu, G D Gamble, G D Mills, J E Garrett, \\ T E Eaton, M I Rees
}

See end of article for authors' affiliations

Correspondence to: Dr R Young, Department of Medicine, Auckland Hospital, Private Bag 92024, Auckland, New Zealand; roberty@adhb. govt.nz

Received 2September 2005 Accepted 6 February 2006 Published Online First 7 February 2006
Background: Chronic obstructive pulmonary disease (COPD) is predominantly the consequence of chronic smoking exposure, but its development may be influenced by genetic variants that affect lung remodelling, inflammation, and defence from oxidant stress. A study was undertaken to determine whether genetic variants within genes encoding the antioxidant enzymes superoxide dismutase (SOD) and catalase may be associated with the development of impaired lung function.

Methods: In a case-control study, the allele and genotype frequencies of functional polymorphisms from SOD1 (CuZnSOD), SOD2 (MnSOD), SOD3 (extracellular SOD), and catalase (CAT) were compared in chronic smokers with normal lung function (resistant smokers) and in those with COPD.

Results: Significantly higher frequencies of the G allele and CG/GG genotype of the 213 SOD3 polymorphism were found in resistant smokers (odds ratios (ORs) 4.3 (95\% $\mathrm{Cl} 1.5$ to 13.3) and $4.2,95 \%$ $\mathrm{Cl} 1.4$ to 13.3), Bonferroni corrected $p=0.02$ and $p=0.02$, respectively) than in those with COPD. There were no differences between the COPD and resistant smokers for the SOD1, SOD2, or CAT polymorphisms tested.

Conclusions: The $213 \mathrm{Gly}$ variant of the SOD3 gene may, through antioxidant or anti-inflammatory effects, confer a degree of resistance in some smokers to the development of COPD.
I is estimated that over $90 \%$ of patients diagnosed with COPD have been chronic smokers, yet only $15-20 \%$ of smokers become symptomatically limited by COPD. ${ }^{1}$ In addition, it has been estimated that only $15 \%$ of the variation in lung function in smokers can be attributed to the degree of smoking exposure ${ }^{2}$ while a little over $40 \%$ is conferred by genetic factors. ${ }^{3}$ Epidemiological studies have consistently shown that some smokers maintain relatively normal lung function even after more than 40 pack years of smokingthat is, they are "resistant" - and these individuals represent about $40-50 \%$ of chronic smokers. ${ }^{25}$ These data, together with family studies, ${ }^{6}$ suggest there is a substantial genetic component to the susceptibility to developing COPD, and suggest that COPD results from chronic smoking exposure in those genetically predisposed to developing impaired lung function.

Given the pathophysiological heterogeneity that exists in COPD, it is not surprising that a number of genetic variants have been implicated. This includes polymorphisms in genes encoding proteins involved in matrix remodelling (such as metalloproteases or their inhibitors), oxidative stress and inflammation (most often cytokines). ${ }^{6}$ We propose that these polymorphisms may confer susceptibility or resistance to chronic smoke exposure either directly or through linkage with functional genetic variants. Such genetic heterogeneity, together with differences in study design, case and/or control selection, small sample sizes and population admixture (or stratification), is likely to account for much of the conflicting findings reported in the COPD literature to date.

Although classical linkage studies have successfully identified genes implicated in Mendelian diseases, this approach has had limited success in complex disorders. This is because diseases of Mendelian inheritance generally involve genetic variants with high penetrance, low population frequency and little, if any, influence from other genetic or environmental factors. In contrast, complex disorders such as COPD have been shown to be strongly linked to the environment through factors such as smoking exposure, occupation, diet, or respiratory tract infections. Not surprisingly, recent linkage studies in pedigrees characterised by poor lung function ${ }^{78}$ suggest that there is a strong gene-environment interaction and linkage to several chromosomal regions have been reported.

There is now growing interest in the use of case-control association studies in complex disorders such as COPD to identify genes of generally high frequency but low penetrance and overall small effects. ${ }^{9-11}$ This may be particularly relevant in COPD where genetic variants may confer either resistance or susceptibility to COPD. Here we report a case-control association study which examined the possible role of candidate polymorphisms in the genes encoding the antioxidant enzymes copper-zinc superoxide dismutase (SOD1), manganese superoxide dismutase (SOD2), extracellular superoxide dismutase (SOD3), and catalase (CAT). Specifically, we have targeted single nucleotide polymorphisms (SNPs) for their likely functional role: SODl +35 A/C which is located adjacent to a splice site, SOD2 Vall6Ala which has been suggested to alter protein structure and function, ${ }^{12}$ SOD3 $213(+760) \mathrm{C} / \mathrm{G}$ which has been shown to alter proteolytic processing, ${ }^{13}$ and catalase $-21 \mathrm{~A} / \mathrm{T}$ which is located in the promoter region just proximal to the start site. ${ }^{14}$ The SOD 3 candidate polymorphism is of particular interest since the $C \rightarrow G$ substitution $(+760)$ leads to an absence of cleaved SOD3 in the serum of carriers in a bimodal distribution. ${ }^{15}{ }^{16}$ Moreover, this genetic variant has been associated with altered antioxidant or anti-inflammatory activity. ${ }^{18}$ Other studies suggest that SOD3 may also influence matrix remodelling ${ }^{19-25}$ in the lung and this may be relevant to the development of COPD. ${ }^{26}$

Abbreviations: COPD, chronic obstructive pulmonary disease; $\mathrm{FEV}_{1}$, forced expiratory volume in 1 second; FVC, forced vital capacity; SNP, single nucleotide polymorphism; SOD, superoxide dismutase 


\section{METHODS}

\section{Subject recruitment}

Group 1: COPD (chronic smokers with impaired lung function consistent with COPD)

We recruited subjects of European descent (all four grandparents European) with COPD who had been previously diagnosed by a specialist physician and who met the following criteria: $>40$ years of age, smoking history of $\geqslant 15$ pack years, forced expiratory volume in 1 second/forced vital capacity $\left(\mathrm{FEV}_{1} / \mathrm{FVC}\right.$ ) ratio $<70 \%$, and $\mathrm{FEV}_{1}<80 \%$ predicted (ERS criteria, ECCS reference values). Pre-bronchodilator spirometric tests were performed according to ATS guidelines. COPD subjects were identified following admission to hospital with an infective exacerbation of COPD or following attendance at a hospital outpatient clinic. They were assessed while in a stable condition with spirometric measurements taken after withholding their usual COPD medications for the following periods: short acting bronchodilators for 6 hours, long acting bronchodilators and inhaled corticosteroids for 12 hours. No subjects had been on oral corticosteroids, inhaled long acting anticholinergic inhalers, or oral bronchodilating agents in the 6 week period prior to testing. Those with a history of any of the following were excluded: childhood or early adulthood asthma, prior evidence of a $>20 \%$ reversibility in $\mathrm{FEV}_{1}$ in response to inhaled bronchodilator treatment, symptoms of breathlessness before 40 years of age, or past lung surgery. COPD subjects were also excluded if they answered yes to the question "Have you ever been diagnosed with bronchiectasis?" or were diagnosed as having bronchiectasis in their hospital records, or on clinical or radiological grounds.

\section{Group 2: Resistant smokers (chronic smokers with near normal lung function)}

We also recruited from the same community (from social clubs for the elderly living in the same city suburbs as the COPD cases) a cohort of resistant smokers of European descent (all four grandparents European) who volunteered to be studied and who met the following criteria: $>40$ years of age, smoking history of $\geqslant 15$ pack years, $\mathrm{FEV}_{1} / \mathrm{FVC}$ ratio of $70-90 \%$, and $\mathrm{FEV}_{1}>80 \%$ predicted.

Using questions from the ATS Respiratory Questionnaire, occupational exposure to dusts and fumes was recorded from the COPD subjects and resistant smokers. Using a PCR based method, ${ }^{27}$ we genotyped the COPD subjects and the resistant smokers for the $\alpha_{1}$-antitrypsin mutations ( $S$ and $Z$ variants) and excluded those with the ZZ or SZ genotype. Those with the MZ genotype were included and made up 5\% of both the COPD and resistant smoker cohorts.

\section{Group 3: Blood donors}

We also recruited 190 blood donors of European descent (all four grandparents European) whose recruitment was unrelated to smoking status and who were healthy with no known respiratory illnesses.

The study was approved by the Auckland ethics committee and all subjects gave informed written consent.

\section{Genotyping}

Genomic DNA was extracted from peripheral blood leucocytes using standard phenol and chloroform methods. Cohorts of patient and control DNA were configured into 96-well polymerase chain reaction (PCR) format containing strategic negative controls. Details of each genotyping assay are shown in table 1. These were modified from previously published methods. ${ }^{14} 28$ PCR amplifications were performed in $25 \mu \mathrm{l}$ reaction volumes containing 50-80 ng genomic DNA, 10 pmol of each primer, $0.2 \mathrm{mM}$ dNTPs, $1 \mathrm{X}$ Qiagen Taq polymerase buffer (containing $15 \mathrm{mM} \mathrm{MgCl}_{2}$ ) and $1 \mathrm{U}$ Qiagen Taq polymerase. Reactions were enhanced with 1 X Qiagen Q solution when required as determined by the PCR optimisation process. The samples were denatured for 5 minutes at $94^{\circ} \mathrm{C}$, subjected to $32-35$ cycles each of 1 minute at $94^{\circ} \mathrm{C}, 1$ minute at $60^{\circ} \mathrm{C}\left(55^{\circ} \mathrm{C}\right.$ for the SODl gene polymorphism), and 1 minute at $72^{\circ} \mathrm{C}$, and elongated for 7 10 minutes at $72^{\circ} \mathrm{C}$ (MJ Research). Aliquots of PCR amplification product were digested for two hours with 5-10 U of restriction enzymes (New England BioLabs) and separated on conventional and molecular screening grade agarose gels (Roche). Digested products were visualised by ultraviolet transillumination following ethidium bromide staining and migration compared against a $\mathrm{l}$ kb plus DNA ladder (Invitrogen) and a positive RFLP control sample.

\section{Statistical analysis}

The demographic characteristics of the COPD and resistant smokers were compared using Fisher's exact test for sex and the Student's $t$ test for unrelated groups for the continuous normally distributed variables. Adjustment for pack years was provided using analysis of covariance. Genotype frequencies were compared with Hardy-Weinberg equilibrium within each cohort. Allele and genotype frequencies were compared between cohorts using the Mantel-Haenszel $\chi^{2}$ test to generate odds ratios with $95 \%$ confidence limits. Logistic regression was used to provide the odds of COPD/resistant smoking for each genotype after adjusting for age and pack years. Analyses were conducted using procedures of SAS Version 9.1 for Windows (SAS Institute Inc). All tests were two tailed and $\mathrm{p}$ values were corrected for number of polymorphisms examined with the Bonferroni method taking $\mathrm{p}<0.05$ as significant. Evidence for population stratification was sought using Structure (v2.1). ${ }^{29}$ Using power calculations we were able to estimate that sample sizes in excess of 200 would be sufficient to detect modest effects with genotype frequencies for the minor allele (approximately $10 \%$ or more) for three of the four polymorphisms at alpha $=0.05$ assuming $80 \%$ power. $^{30}$

Table 1 Polymerase chain reaction primers, restriction enzymes, and separation conditions for genotyping

\begin{tabular}{|c|c|c|c|}
\hline Gene (polymorphism) & Primer sequence & $\begin{array}{l}\text { Restriction enzyme } \\
\text { (Temperature) }\end{array}$ & Separation conditions \\
\hline SODI ( $\rightarrow$ $\rightarrow$ C, +35 exon3/intron3) & $\begin{array}{l}\text { Forward: 5'CTATCCAGAAAACACGGTGGGCC3' } \\
\text { Reverse: 5'TCTATATTCAATCAAATGCTACAAAACC3' }\end{array}$ & Hha I $\left(37^{\circ} \mathrm{C}\right)$ & $2 \%$ agarose \\
\hline SOD2 (Val16Ala, $T \rightarrow C$ ) & $\begin{array}{l}\text { Forward: 5'CGCAGCCCAGCCGTGCGTA3' } \\
\text { Reverse: 5'GTGAGGTCCAGGGCGCCGT3' }\end{array}$ & Bsa WI $\left(60^{\circ} \mathrm{C}\right)$ & $2 \%$ agarose \\
\hline SOD3 (Arg213Gly, A $\rightarrow$ G) & $\begin{array}{l}\text { Forward: 5'GCAACCAGGCCAGCGTGGAGAACGGGAA3' } \\
\text { Reverse: 5'CCAGAGGAGAAGCTCAAAGGCAGA3' }\end{array}$ & Mwo I $\left(60^{\circ} \mathrm{C}\right)$ & $3 \% \mathrm{MS}$ agarose \\
\hline Catalase ( $\mathrm{A} \rightarrow \mathrm{T}$, promoter) & $\begin{array}{l}\text { Forward: 5'AATCAGAAGGCAGTCCTCCC3' } \\
\text { Reverse: 5'TCGGGGAGCACAGAGTGTAC3' }\end{array}$ & $\operatorname{Hinf} \mathrm{I}\left(37^{\circ} \mathrm{C}\right)$ & $2 \%$ agarose \\
\hline
\end{tabular}


Table 2 Mean (SD) characteristics of the COPD cohorts and resistant smokers

\begin{tabular}{|c|c|c|c|}
\hline Parameter & $\begin{array}{l}\text { COPD } \\
(\mathrm{N}=230)\end{array}$ & $\begin{array}{l}\text { Resistant smokers } \\
(\mathrm{N}=210)\end{array}$ & $\begin{array}{l}\text { Differences } \\
\text { (p value) }\end{array}$ \\
\hline $\begin{array}{l}\text { \% men } \\
\text { Age (years) } \\
\text { Pack years } \\
\text { Cigarettes/day } \\
\mathrm{FEV}_{1} \text { (I) } \\
\mathrm{FEV}_{1} \text { (\% predicted) } \\
\mathrm{FEV}_{1} / \mathrm{FVC}\end{array}$ & $\begin{array}{l}59 \% \\
65(10) \\
50(29) \\
24(15) \\
1.6(0.56) \\
42(18) \\
51(15)\end{array}$ & $\begin{array}{l}63 \% \\
59(13) \\
42(25) \\
24(12) \\
2.9(0.73) \\
96 \%(10) \\
82(8)\end{array}$ & $\begin{array}{l}0.88 \\
<0.001 \\
0.002 \dagger \\
0.76^{*} \\
0.01 \\
<0.001 \\
<0.001\end{array}$ \\
\hline
\end{tabular}

\section{RESULTS}

\section{Subjects}

The characteristics of the COPD and resistant smoker groups are summarised in table 2. Consistent with our criteria for recruitment of resistant smokers, lung function in this group was near normal and significantly greater than the spirometric means of the COPD group. In the blood donor cohort $63 \%$ were men and their mean (SD) age was 50 (10) years. At the time of recruitment $63 \%$ had never smoked, $30 \%$ were exsmokers, and $7 \%$ were current smokers. Information on lung function was not available in this group but none had a diagnosis of COPD.

The COPD and resistant smokers had the same mean daily cigarette consumption $(24 /$ day, $p=0.76)$, comparable age of smoking onset (17 and 16 years respectively, $\mathrm{p}=0.55)$, comparable age when stopped smoking ( 58 and 51 years respectively, $\mathrm{p}=0.12$ ), although the resistant smokers were younger $(59 \vee 65$ years, $\mathrm{p}<0.001)$ with a lower pack year history ( $42 \vee 50$ pack years, $\mathrm{p}=0.002$ ) than smokers with COPD. When the pack years were adjusted for age and the same daily cigarette consumption allowed for, the smoking exposures were not statistically different between the two groups. We found no evidence for population stratification between resistant smokers and those with COPD using 40 unlinked SNPs from different genes (mean $\chi^{2}=3.1$, $\mathrm{p}=0.61)$.

We estimate that our analyses, which included a burn-in period of 10000 iterations to collect data for 10000 iterations, had a power of more than $97 \%$ to detect $(\mathrm{p}<0.05)$ population stratification (median $\left.\mathrm{p}=3.3 \times 10^{-5}\right)$. The SNPs were part of a panel derived for the purposes of a case association study for coronary artery disease and were not known to be associated with either of our smoking phenotypes.

\section{Allele and genotype frequency comparisons}

The completion rate for all four genotypes was over $97 \%$ and all were in Hardy-Weinberg equilibrium. The completion rate for $\alpha_{1}$-antitrypsin genotypes was $100 \%$, and those with the $\mathrm{MZ}$ genotype were included making up 5\% of both the COPD and resistant smoker cohorts. Any subject with the ZZ genotype was excluded and there were no SZ subjects. Statistical differences between allele or genotype frequencies were found for the SOD1 (intron 3), SOD2 (Vall6Ala), and CAT promoter polymorphisms (table 3 ).

The only significant difference between the groups was for the SOD3 Arg213Gly polymorphism (table 4). To ensure accuracy of the method, genotyping of this polymorphism was repeated in all three cohorts using the same RFLP method. We found a $2 \%$ error rate and assigned genotype based on the results of a third assay for those genotypes discordant from the first two assays. Resistant smokers had a significantly higher $\mathrm{G}$ allele frequency $(5 \%$ and $1 \%$ respectively, OR 4.3, p<0.05) and a significantly higher CG/GG genotype frequency than subjects with COPD (9\% and $2 \%$ respectively, OR $4.2, \mathrm{p}<0.05)$. Combining the $\mathrm{CG}$ and $\mathrm{GG}$ genotypes can be justified on an a priori basis as both are associated with increased serum levels of SOD3. ${ }^{15}$ When an older subgroup ( $\geqslant 55$ years) of 137 resistant smokers was examined, they matched the COPD subjects more closely (mean (SD) age 67 (8) years and mean (SD) pack years 48 (22)). In this comparison, higher $G$ allele and CG/GG genotype frequencies were still evident in the resistant smokers (OR 4.2 and 3.8 respectively, uncorrected $\mathrm{p}=0.01$ and 0.02 respectively). A comparison of younger COPD patients with the resistant smokers did not change the above findings (data not shown). No subject with the MZ genotype were carriers of the SOD3 G allele so, after excluding the former, there was still a significant excess of the SOD $213 \mathrm{G}$ allele in the resistant smokers compared with those with COPD.

After adjustment for age, sex and smoking history, logistic regression of all COPD subjects and resistant smokers showed that the odds of being a resistant smoker compared with having COPD modelled for the CG/GG genotype versus the CC genotype was 6.1 (95\% CI 1 to 38 ), $p=0.05$. The blood

Table 3 SOD1, SOD2 and catalase polymorphism allele and genotype frequencies in COPD patients and resistant smokers

\begin{tabular}{|c|c|c|c|c|c|}
\hline \multirow{2}{*}{$\begin{array}{l}\text { Polymorphism } \\
\text { SOD1-intron } 3 \text { SNP }\end{array}$} & \multicolumn{2}{|c|}{ Allele frequencies (\%) } & \multicolumn{3}{|c|}{ Genotype frequencies (\%) } \\
\hline & $A$ & C & $\mathrm{AA}$ & $\mathrm{AC}$ & $\mathrm{CC}$ \\
\hline COPD & 95 & 5 & 92 & 7 & 1 \\
\hline Resistant smokers & 94 & 6 & 90 & 9 & 1 \\
\hline SOD2-Val16Ala & $T$ & C & $\pi$ & TC & $\mathrm{CC}$ \\
\hline COPD & 49 & 51 & 23 & 52 & 25 \\
\hline Resistant smokers & 49 & 51 & 22 & 54 & 24 \\
\hline Catalase-promoter SNP & $\mathrm{T}$ & $A$ & $\Pi \pi$ & TA & $\mathrm{AA}$ \\
\hline COPD & 28 & 72 & 7 & 41 & 52 \\
\hline Resistant smokers & 31 & 69 & 10 & 42 & 48 \\
\hline
\end{tabular}

No significant differences were found for either the allele or genotype (absolute) frequencies for all three polymorphisms. 


\begin{tabular}{|c|c|c|c|c|c|}
\hline \multirow[b]{2}{*}{ Groups (\% genotyped) } & \multicolumn{2}{|c|}{ Allele frequencies (\%) } & \multicolumn{3}{|c|}{ Genotype frequencies $(\%)$} \\
\hline & c & G & CC & CG & GG \\
\hline $\begin{array}{l}\text { Smokers with COPD } \\
\mathrm{N}=222(97 \% \text { of total })\end{array}$ & $439(99 \%)$ & $5(1 \%)$ & $217(98 \%)$ & $5(2 \%)$ & $0(0 \%)$ \\
\hline $\begin{array}{l}\text { Resistant smokers } \\
N=203 \text { (97\% of total) }\end{array}$ & $387(95 \%)$ & $19(5 \%)$ & 185 (91\%) & $17(8 \%)$ & $1(1 \%)$ \\
\hline $\begin{array}{l}\text { Blood donor controls } \\
\mathrm{N}=189 \text { ( } 99 \% \text { of total) }\end{array}$ & $369(98 \%)$ & $9(2 \%)$ & $182(96 \%)$ & $5(3 \%)$ & $2(1 \%)$ \\
\hline \multicolumn{6}{|c|}{$\begin{array}{l}\text { Allele frequency: } G v C \text { for resistant smokers compared with the COPD group: OR } 4.3(95 \% \mathrm{Cl} 1.5 \text { to } 13.3 \text { ), } \\
\chi^{2}=8.6 \text {; uncorrected } p=0.004 \text { (Bonferroni corrected } p=0.016 \text { ). } \\
\text { Genotype frequency: CG/GG (co-dominant model) } v \text { CC for resistant smokers compared with the COPD group: } \\
\text { OR } 4.2,95 \% \mathrm{Cl} 1.4 \text { to } 13.3 \text { ), } \chi^{2}=7.8 \text {; uncorrected } p=0.005 \text { (Bonferroni corrected } p=0.02 \text { ). }\end{array}$} \\
\hline
\end{tabular}

donor controls, although younger, represent a relatively unselected and ethnically matched cohort. With the limited sample size, they provide a second group from which to establish the allele and genotype frequencies in our general population. These were comparable to those reported in other studies. ${ }^{141631}$ The allele and genotype frequencies for the SOD 3 variant in the blood donor group were similar to those of the COPD cohort (despite the age difference) and, again, less than that in the resistant smokers $(p=0.04$ and $p=0.08$ for genotype and allele frequencies, respectively). This supports our view that the $G$ allele is found more frequently in resistant smokers rather than being less frequent in smokers with COPD - that is, the G allele may confer resistance to impaired lung function as suggested by its functional effects. Adjustment for occupational exposure to dust and fumes did not alter the findings of the logistic regression analysis.

\section{DISCUSSION}

Using a case-control study, we have examined whether there is an association between genetic variants of antioxidant polymorphisms and susceptibility or resistance to COPD from smoking. Our results suggest that the 213 Gly SOD 3 genetic variant is associated with conferring resistance to COPD in some smokers. We believe this observation was made possible, in part, by using a case-control study design which compared COPD patients and smokers with near normal lung function matched for sex, ethnicity, city suburb, and smoking exposure. This approach is based on three important epidemiological studies that collectively show that (1) some smokers maintain normal lung function despite decades of heavy smoking exposure; (2) non-smokers and light smokers $(<20$ pack years $)$ have a normal distribution in lung function; and ( 3 ) when the smoking exposure dose exceeds about 30-40 pack years, the separation of smokers into those resistant to smoking (in terms of normal lung function) and those with impaired lung function (consistent with COPD) becomes quite distinct. ${ }^{245}$ We found no evidence of population stratification in our smoking cohorts and our genotype/ allele frequencies were in Hardy-Weinberg equilibrium. Although a false positive result from sampling bias and/or confounding (such as survival effect) is still possible, we believe this is less likely given that the genetic frequencies were no different between blood donors and COPD subjects, in contrast to resistant smokers. We believe that, regardless of the size of the study cohort or appropriate correction for multiple comparisons, replication of our result in other populations best substantiates our findings. In this regard, a small case-control study in the United States and a larger population study in Denmark have recently reported similar findings. ${ }^{32}{ }^{33}$ We conclude that the excess of the 213 Gly SOD3 genetic variant in resistant smokers is of potential relevance.

There are now considerable data to support the hypothesis that SOD3 functions as an important antioxidant or antiinflammatory protein in smokers and that the functional genetic variant of the SOD3 gene may confer protection from the adverse affects of cigarette smoke on the lung. Firstly, SOD3 has been shown to be strongly localised in lung tissue ${ }^{18}$ where it is synthesised by type II pneumocytes tightly bound to extracellular lung matrix ${ }^{20}$ and functions to protect the lung. ${ }^{21}$ Secondly, in a transgenic mouse model, overexpression of SOD3 in the lungs resulted in markedly attenuated oxidant mediated lung damage primarily through reducing the recruitment of neutrophils. ${ }^{22}$ Lastly, the 213 Gly variant of the SOD3 polymorphism has been shown to account almost exclusively for the high serum SOD3 activity (those with the CG or GG genotpye), which segregates as a bimodal distribution in the general population and confers a tenfold or greater antioxidant activity. ${ }^{15}$

The mechanism by which the $213 \mathrm{Gly}$ variant confers this protection comes from studies showing it alters the heparin binding site of the SOD3 protein reducing its affinity for the extracellular matrix and increasing its half life by reducing normal proteolytic cleavage. ${ }^{17}$ It has been proposed that the secretion of uncleaved SOD3 (generated by the 213Gly variant) may result in a greater availability of SOD3 to provide an antioxidant or anti-inflammatory response. ${ }^{17} 22$ However, it should be noted that a deleterious effect by reducing bound SOD3 to extracellular matrix has also been suggested. ${ }^{15}$ We believe that the significant excess of the 213Gly variant in resistant smokers compared with blood donors and smokers with COPD is consistent with the protective effects described above. Specifically, the $213 \mathrm{Gly}$ variant may confer a degree of protection from the oxidant or inflammatory challenge from smoking through one or more of the following: (1) resistance to cleavage, (2) prolonging tissue binding, and/or (3) reducing neutrophil influx. As only $9 \%$ of our resistant cohort carried a copy of this variant, other protective genes will be implicated as discussed below. The presence or absence of this genetic variant therefore contributes to determining how smoking affects lung function in some smokers. We propose that the protective effect of the $213 \mathrm{Gly}$ genetic variant is especially relevant to people with a smoking history where a gene-environment interaction exists. This is supported by the Danish study ${ }^{33}$ where the protective effect of the $213 \mathrm{Gly}$ variant was confined to smokers. Given these observations, it is perhaps not surprising that this genetic variant was not found to be associated with asthma where smoking is much less prevalent and different pathological processes are implicated. ${ }^{31}$ Of interest is the results of a genome wide scan of 
pulmonary function (specifically $\mathrm{FEV}_{1} / \mathrm{FVC}$ ) that identified a locus on chromosome 4 (D4S403 and D4S1511), a region that includes the gene encoding extracellular SOD (chromosome $4 \mathrm{pl} 6.3) .^{34}$

We have previously described, in a subset of the cohorts used in this study, a functional polymorphism in the gene encoding transforming growth factor- $\beta_{1}$, another protein thought to modulate inflammatory processes in the lung that also appears to confer a protective effect in smokers. ${ }^{35}$ These two findings support our hypothesis that resistance or susceptibility to smoking induced decline in lung function is probably the result of many genetic variants that confer either a protective or pathological phenotype leading to resistance or COPD, respectively. Because of the modest sample size and limited genotyping, the role of SOD1, SOD2, and catalase on lung function in chronic smokers cannot be ruled out by this study, although it is interesting that they function primarily as intracellular rather than extracellular enzymes in the antioxidant pathway.

Although the 213 Gly polymorphism is functional and it is plausible that it influences the development of COPD as discussed above, ${ }^{26}{ }^{32-34}$ we cannot confirm a directly causal role. Similarly, we cannot rule out whether other genetic variants in linkage disequilibrium with this polymorphism are of functional importance. However, some support for a direct role for the SOD3 213 variant comes from transgenic mouse studies where overexpression of SOD3 attenuates the lung tissue damage from air pollutants, ${ }^{36}$ particularly from heavy metals found in cigarette smoke previously linked to smokers with emphysema. ${ }^{37}$

In conclusion, we report the novel association between a functional genetic variant of the SOD3 gene and resistance to COPD. There are substantive data to implicate this polymorphism in lung development, lung function, and response to oxidant load. In the current study we believe we have addressed many of the concerns surrounding case association studies. $^{38}$ Specifically we have (1) achieved sample sizes sufficiently powered to detect large differences, (2) prioritised our SNP selection to functional variants in implicated candidate genes, (3) excluded population stratification by accepted methods, (4) corrected for multiple testing, (5) avoided sub-analysis to generate associations, and (6) compared cases with a control group well matched for confounding variables and derived from the same ethnic, socioeconomic, and geographical region. Despite this, we believe replication in other cohorts is required to validate associations such as this. To this end, we and others ${ }^{32}{ }^{33}$ have preliminary results that support our findings that the SOD3 $213 \mathrm{G}$ allele appears to confer a protective effect from impaired lung function in smokers. To further confirm this we are undertaking a second larger community based study to test this association in greater detail. We conclude that, inherent in the complex nature of the genetics of COPD, genotyping a diverse range of genetic variants may be necessary in order to better understand the contributing effects of a number of genes which probably confer weak effects only.

\section{ACKNOWLEDGEMENTS}

The authors thank all participants, in particular those recruited through clubrooms for local Returned Services Associations, for their kind help in participating in this study, and Mr Zoran Ljubisavljevic for his help with computing and data storage.

\section{Authors' affiliations}

R P Young, P N Black, C Eddy, L Wu, G D Gamble, Department of Medicine, University of Auckland, Auckland, New Zealand

R Hopkins, T E Eaton, Respiratory Services, Green Lane Hospital, Auckland, New Zealand
J E Garrett, Department of Respiratory Medicine, Middlemore Hospital, South Auckland, New Zealand

G D Mills, Department of Respiratory Medicine, Waikato Hospital, Hamilton, New Zealand

M I Rees, School of Medicine, University of Wales Swansea Singleton Park, Swansea, UK

This study was supported by grants from the University of Auckland, Lottery Grants of New Zealand, Auckland Medical Research Foundation, Paykel Trust and Health Research Council of New Zealand.

Competing interests: none declared.

\section{REFERENCES}

1 Buist SA. Smoking and other risk factors. In: Murray JF, Nadel JA, eds. Textbook of respiratory medicine. Philadelphia: WB Saunders, 1988:1001-29.

2 Burrows B, et al. Quantitative relationship between cigarette smoking and ventilatory function. Am Rev Respir Dis 1977;115:195-205.

3 Coultas DB, Hanis C, Howard CA, et al. Heritability of ventilatory function in smoking and non-smoking New Mexico Hispanics. Am Rev Respir Dis $1991 ; 144: 770-5$.

4 Fletcher $\mathrm{C}$, Peto R. The natural history of chronic airflow obstruction. BMJ 1977;1:1645-72.

5 Dockery DW, Speizer FE, Ferris BG, et al. Cumulative and reversible effects of lifetime smoking on simple lung function in adults. Am Rev Respir Dis 1988; 137:286-92.

6 Sandford AJ, Weir TD, Pare PD. Genetic risk factors for chronic obstructive pulmonary disease. Eur Respir J 1997;10:1380-91.

7 Silverman EK. Genetic epidemiology of COPD. Chest 2002;121:1-6S.

8 Joost O, Wilk JB, Cupples LA, et al. Genetic loci influencing lung function: a genome wide scan in the Framingham study. Am J Respir Crit Care Med 2002; 165:795-9.

9 Risch N, Merikangas K. The future of genetic studies of complex human diseases. Science 1996;273:1516-7.

10 Cardon LR, Bell Jl. Association study designs for complex diseases. Nat Rev Genet 2001;2:91-9.

11 Tabor HK, Risch NJ, Myers RM. Candidate-gene approaches for studying complex genetic traits: practical considerations. Nat Rev Genet 2002;3:1-7.

12 Wang LI, Miller DP, Sai Y, et al. Manganese superoxide dismutase alanine-tovaline polymorphism at codon 16 and lung cancer. J Natl Cancer Int 2001;93:1818-21.

13 Olsen DA, Petersen SV, Oury TD, et al. The intracellular processing of extracellular dismutase (EC-SOD) is a two step event. J Biol Chem 2004;279:22152-7.

14 Ukkola O, Erkkila PH, Savolainen MJ, et al. Lack of association between polymorphisms of catalase, copper-zinc superoxide dismutase (SOD), extracellular SOD and endothelial nitric oxide synthase genes and macroagiopathy in patients with type 2 diabetes mellitus. J Intern Med 2001;249:451-9.

15 Sandstrom J, Nilsson P, Karlsson K, et al. 10-fold increase in human plasma extracellular superoxide dismutase content caused by a mutation in heparinbinding domain. J Biol Chem 1994;269:19163-6.

16 Marklund SL, Nilsson P, Israelsson K, et al. Two variants of extra-cellular superoxide dismutatse: relationship to cardiovascular risk factors in an unselected middle-aged population. J Intern Med 1997;242:5-14.

17 Bowler RP, Nicks M, Olsen DA, et al. Furin proteolytically processes the heparin binding region of extracellular superoxide dismutase. J Biol Chem 2002;277:16505-11.

18 Fattman CL, Chu CT, Kulich SM, et al. Altered expression of extracellular superoxide dismutase in mouse lung after bleomycin treatment. Free Radic Biol Med 2001;31:1198-207.

19 Marklund SL. Extracellular superoxide dismutase in human tissues and human cell lines. J Clin Invest 1984;74:1398-403.

20 Su WY, Folz R, Chen JS, et al. Extracellaur superoxide dismutase mRNA expressions in the human lung by in situ hybridisation. Am J Respir Cell Mol Biol 1997; 16:162-70.

21 Loenders B, Van Mechelen E, Nicolai S, et al. Localisation of extracellular superoxide dismutase in rat lung: neutrophils and macrophages as carriers of the enzyme. Free Radic Biol Med 1998;24:1097-106.

22 Folz RJ, AbushammaAM, Suliman HB. Extracellular superoxide dismutase in the airways of transgenic mice reduces inflammation and attenuates lung toxicity following hyperoxia. J Clin Invest 1999;103:1055-66.

23 Oury TD, Schaefer LM, Fattman CL, et al. Depletion of pulmonary EC-SOD after exposure to hyperoxia. Am J Physiol - Lung Cell Mol Physiol 2002;283:L777-84.

24 Kurys E, Kurys P, Kuzniar A, et al. Analysis of antioxidant enzyme activity and magnesium level in chronic obstructive pulmonary disease. Annal Univers Masriae Curie-Sklodoaska 2001;56:261-6.

25 Matskevich GN, Korotrina RN, Devlikanova AS, et al. The study of the antioxidant enzymes in erythrocytes in lung disease. Patolog Fiziol Eksper Tera 2003;2:2-25.

26 Kinuula VL, Crapo JD. Superoxide dismutases in the lung and human lung diseases. Am J Respir Crit Care Med 2003;167:1600-19.

27 Sandford AJ, Chagani T, Spinelli JJ, et al. Alpha-1-antitrypsin genotypes and the acute phase response to open heart surgery. Am J Respir Crit Care Med 1999;159:1624-8. 
28 Kimura K, Isashiki Y, Sonoda S, et al. Genetic association of manganese superoxide dismutase with exudative age-related macular degeneration. Am J Ophthalmol 2000;130:769-73

29 Pritchard JK, Stephens M, Donnelly P. Inference of population structure from multilocus genotype data. Genetics 2000;155:945-59.

30 Purcell S, Cherny SS, Sham PC. Genetic power calculations: design of linkage and association genetic mapping studies of complex traits. Bioinformatics 2003; 19:149-50

31 Kinnula VL, Lehtonen $S$, Koisteinen $P$, et al. Two functional variants of the superoxide dismutase genes in Finnish families with asthma. Thorax 2004;59:116-9.

32 Bowler RP, Wheeler C, Duda B, et al. Polymorphisms in the extracellular superoxide dismutase gene and plasma EC-SOD concentrations are associated with a decreased risk of COPD. Thomas L Petty Aspen Lung Conference, June, 2005.
33 Juul K, Tybjaerg-Hansen A, Marklund S, et al. Genetically increased antioxidative protection and decreased chronic obstructive pulmonary disease. Am J Respir Crit Care Med 2006 (in press).

34 Wilk JB, Destefano AL, Arnett DK, et al. A genome-wide scan of pulmonary function measures in the Heart, Lung and Blood Institute family heart study. Am J Respir Crit Care Med 2003;167:556-61.

35 Wu L, Chau J, Young RP, et al. Transforming growth factor- $\beta 1$ genotype and susceptibility to chronic obstructive pulmonary disease. Thorax 2004;59:126-9.

36 Ghio AJ, Suliman HB, Carter JD, et al. Overexpression of extracellular superoxide dismutase decreases lung injury after exposure to oil fly ash. Am J Physiol Lung Cell Mol Physiol 2002;283:L21 1-8.

37 Hendrik DJ. Smoking, cadmium and emphysema. Thorax 2004;59:184-5.

38 Hall IP, Blakey JD. Genetic association studies in Thorax. Thorax 2005;60:357-9.

\section{LUNG ALERT}

Non-invasive ventilation improves survival in patients with motor neuron disease

$\Delta$ Bourke SC, Tomlinson M, Williams TL, et al. Effects of non-invasive ventilation on survival and quality of life in patients with amyotrophic lateral sclerosis: a randomised controlled trial. Lancet Neurol 2006;5:140-7

T

his randomised controlled trial allocated patients with amyotrophic lateral sclerosis to either standard care $(\mathrm{n}=19)$ or non-invasive ventilation (NIV, $\mathrm{n}=22$ ). Patients were randomised when they developed orthopnoea with a maximum inspiratory pressure of $<60 \%$ or symptomatic daytime hypercapnia. They were assessed with the SF-36 and sleep apnoea quality of life index (SAQLI) scores. The authors found that quality of life scores were maintained at 75\% above baseline for longer in the patients randomised to NIV (192 v 46 days, $\mathrm{p}=0.0013)$. Furthermore, in the patients with good bulbar function there was a median survival benefit of 205 days $(p=0.006)$. The subgroup with poor bulbar function had improved sleep related symptoms but no survival benefit.

This study confirms that, rather than prolonging suffering, NIV extends survival in motor neuron disease with improved quality of life in those with good bulbar function, and improves sleep symptoms in those with poor bulbar function.

A McKendrick

SHO, Kettering General Hospital, UK; avance@doctors.net.uk 